\title{
Axial anomaly in very special relativity
}

\author{
Jorge Alfaro $\odot$ \\ Instituto de Física, Pontificia Universidad Católica de Chile, \\ Avenida Vicuña Mackenna 4860, Santiago, Chile
}

(Received 27 December 2020; accepted 3 March 2021; published 12 April 2021)

\begin{abstract}
In this paper we study the axial anomaly in very special relativity electrodynamics using Pauli-Villars and dimensional regularization of ultraviolet divergences and Mandelstam-Leibbrandt regularization of infrared divergences. We compute the anomaly in 2 and 4 dimensional space-time. We find that this procedure preserves the vector Ward identity(charge conservation) and reproduce the standard axial anomaly in 2 and 4 dimensions without corrections from VSR. Finally, we show how to obtain the anomaly in the path integral approach.
\end{abstract}

DOI: 10.1103/PhysRevD.103.075011

\section{INTRODUCTION}

The Standard Model of particle physics (SM) is a very successful theory. With the discovery of the Higgs boson at CERN, its particle composition was completed [1].

But the discovery of neutrino oscillations showed that the neutrinos have mass whereas in the SM they are massless [2].

One of the most important problems of particle physics is to provide a mass for the neutrino without disturbing the chiral nature of the SM, since neutrinos appear to be left handed.

The seesaw mechanism is a popular mechanism to obtain massive neutrinos [3]. However, it means to introduce new particles and new interactions.

One possibility to have massive chiral neutrinos is very special relativity(VSR) [4]

VSR assumes that the true symmetry of Nature is not the full Lorentz group, but some of its subgroups. The most interesting of these subgoups are $\operatorname{Sim}(2)$ and $\operatorname{Hom}(2)$. Using these subgroups new terms are allowed such that the neutrino get a mass [5].

Various applications of VSR have been considered, like the inclusion of supersymmetry [6,7], curved spaces [8,9], noncommutativity [10,11], dark matter [12], and also in cosmology [13].

Some time ago, we proposed the SM with VSR [14] (VSRSM). Its particle composition and interactions are the same as in the SM, but neutrinos can have a VSR mass without lepton number violation.

Published by the American Physical Society under the terms of the Creative Commons Attribution 4.0 International license. Further distribution of this work must maintain attribution to the author(s) and the published article's title, journal citation, and DOI. Funded by SCOAP.
Loop computations in VSR are nontrivial though. New infrared divergences appear and they have to be regularized. We studied how to do so using the calculation of integrals in the Mandelstam-Leibbrandt (ML) prescription $[15,16]$ introduced in [17], in [18,19]. The Ward identities corresponding to the gauge and the $\operatorname{Sim}(2)$ symmetry of the model are preserved.

Last year, we applied these techniques to the Schwinger model in VSR [20] and to the photon mass in VSR [21].

A very important test that the VSRSM has to pass is the cancellation of axial anomalies. Being a chiral local gauge symmetry model, the presence of chiral anomalies may kill the model, because the gauge symmetry will be lost and renormalizability and unitarity could not be simultaneously satisfied.

In [20] we did a computation of the two dimensional axial anomaly. We obtained that the vector current is conserved and the axial anomaly get a correction from VSR in the form of a multiplicative factor.

The authors of [22] tried to compute the axial anomaly in four dimensions using the prescription to treat $\gamma^{5}$ introduced in [23]. They claim that there is an anomaly in the vector current as well as in the axial vector current. However their computation missed two important graphs. (Please see Chap. IV).

In this paper we study the axial anomaly in two and four dimensions using Pauli-Villars (PV) and dimensional(DR) regularization of ultraviolet divergences and ML prescription for infrared divergences. We show explicitly that the vector current is conserved and that the axial anomaly is the same we get in Lorentz invariant electrodynamics, without any correction from VSR. Our result relies on two properties of the ML prescription: First, it allows shifting of the loop momentum variable (which implies gauge invariance) and second, it respects naive power counting. 
According to this result, the VSRSM must be free from anomalies and therefore consistent.

In the VSR gauge field action a very interesting possibility opens up: A gauge invariant mass term for the gauge field $[14,24,25]$. In the present work, we did not include such a mass term for the photon because it will not affect the axial anomaly, since the axial anomaly is due to a loop of fermions.

The paper is written as follows. In Sec. II we define the lagrangian of VSR electrodynamics and derive the Feynman rules that will be used to compute the anomalies. In Sec. III we compute the axial anomaly in two dimensional space time. In Sec. IV we study the axial anomaly in four dimensions. In Sec. V we study the axial anomaly in $2 d$ using DR. In Sec. VI, we derive the axial anomaly in 4d, using DR. In Sec. VII we present the derivation of the axial anomaly using the path integral. Finally in Sec. VIII we draw some conclusions.

\section{THE MODEL}

The electrodynamics sector of the VSRSM in the Feynman gauge.

$$
\begin{aligned}
\mathcal{L}= & \bar{\psi}\left(i\left(D+\frac{1}{2} \not h m^{2}(n \cdot D)^{-1}\right)-M\right) \psi \\
& -\frac{1}{4} F_{\mu \nu} F^{\mu \nu}-\frac{\left(\partial_{\mu} A_{\mu}\right)^{2}}{4} \\
D_{\mu}= & \partial_{\mu}-i e A_{\mu}, \quad F_{\mu \nu}=\partial_{\mu} A_{\nu}-\partial_{\nu} A_{\mu}
\end{aligned}
$$

The vector current (electric charge conservation) is

$$
j^{\mu}=\bar{\psi} \gamma^{\mu} \psi+\frac{1}{2} m^{2}\left(\frac{1}{n \cdot D^{\dagger}} \bar{\psi}\right) \not h n^{\mu}\left(\frac{1}{n \cdot D} \psi\right)
$$

The axial vector current is

$$
j^{\mu 5}=\bar{\psi} \gamma^{\mu} \gamma^{5} \psi+\frac{1}{2} m^{2}\left(\frac{1}{n \cdot D^{\dagger}} \bar{\psi}\right) \not n n^{\mu} \gamma^{5}\left(\frac{1}{n \cdot D} \psi\right)
$$

Both currents are conserved at the classical level [20]. We are interested in computing expectation values of these currents.

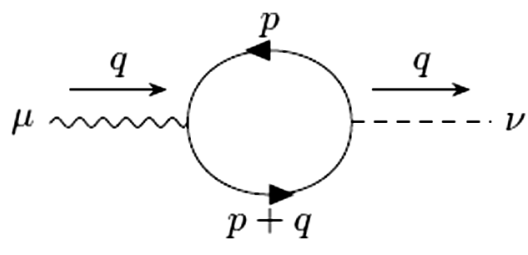

FIG. 1. $i \Pi^{15 \mu \nu}$ (Eq. (3) ).

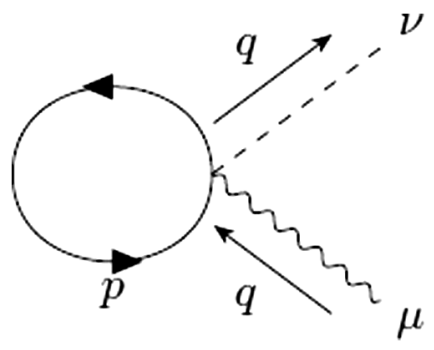

FIG. 2. $i \Pi^{25 \mu \nu}$ (Eq. (4)).

To get the Feynman rules we use the expansion of $(n \cdot D)^{-1}$ both in the currents and the Lagrangian.

$$
\begin{aligned}
(n \cdot D)^{-1}= & \left(1+i e(n . \partial)^{-1}(n . A)\right. \\
& +(i e)^{2}(n . \partial)^{-1}(n . A)(n . \partial)^{-1}(n . A) \\
& +(i e)^{3}(n . \partial)^{-1}(n . A)(n . \partial)^{-1}(n . A) \\
& \left.\times(n . \partial)^{-1}(n . A)\right)(n . \partial)^{-1}+\cdots
\end{aligned}
$$

The Feynman rules are listed in Appendix A.

\section{TWO DIMENSIONAL AXIAL ANOMALY}

In this case we have to compute the expectation value of the axial vector current in a background field $A_{\nu}$. We use the convention of [26], $\epsilon^{01}=+1$.

$$
\left\langle j^{5 \nu}(q)\right\rangle=\int d^{2} x\left\langle j^{5 \nu}(x)\right\rangle e^{i q x}=(-i e)^{-1} i \Pi^{5 \mu \nu}(q) A_{\mu}
$$

The contribution to the two dimensional anomaly in VSR electrodynamics is given by the two graphs (Figs. 1 and 2):

$$
\begin{aligned}
i \Pi^{15 \mu \nu}= & -(-i e)^{2} \int d p \operatorname{Tr}\left\{\left[\gamma^{\mu}+\frac{1}{2} n^{\mu}(\not h) m^{2}(n \cdot(p+q))^{-1}(n \cdot p)^{-1}\right]\right. \\
& \left.\times \frac{i\left(\not p+M-\frac{m^{2}}{2} \frac{\not}{n \cdot p}\right)}{p^{2}-M^{2}-m^{2}+i \varepsilon}\left[\gamma^{\nu}+\frac{1}{2} n^{\nu}(\not h) m^{2}(n \cdot(p+q))^{-1}(n \cdot p)^{-1}\right] \gamma^{5} \frac{\left.i(\not p+\not q)+M-\frac{m^{2}}{2} \frac{\not}{n \cdot(p+q)}\right)}{(p+q)^{2}-M^{2}-m^{2}+i \varepsilon}\right\}
\end{aligned}
$$


$i \Pi^{25 \mu \nu}=(-1)(i e)^{2} n^{\mu} n^{\nu} i \int d p(n \cdot p)^{-1}(n \cdot p)^{-1}\left[(n \cdot(q+p))^{-1}+(n \cdot(-q+p))^{-1}\right] \operatorname{Tr}\left\{\frac{1}{2} \not h m^{2} \frac{i\left(\not p+M-\frac{m^{2}}{2} \frac{\not p}{n \cdot p}\right)}{p^{2}-M^{2}-m^{2}+i \varepsilon} \gamma^{5}\right\}$.

To compute the axial anomaly we will use Pauli-Villars regularization and Mandelstam-Leibbrandt prescription to treat infrared divergences. We will follow Ref. [27].

Notice that Eq. (3) is logarithmically divergent and Eq. (4) is finite.

It is easy to check that formally:

$$
q_{\mu}\left(\Pi^{15 \mu \nu}+\Pi^{25 \mu \nu}\right)=0
$$

if shift of the integration variable $p \rightarrow p+k$ is allowed. Here $k$ is a constant vector. This would be true if the integral (3) would be finite.

Introduce a Pauli-Villars particle of mass $\bar{M}$ and define the regularized amplitude:

$$
\Pi^{5 R \mu \nu}(M, \bar{M}, q)=\Pi^{15 \mu \nu}(M, q)+\Pi^{25 \mu \nu}(M, q)-\Pi^{15 \mu \nu}(\bar{M}, q)-\Pi^{25 \mu \nu}(\bar{M}, q) .
$$

Since $\Pi^{5 R \mu \nu}(M, \bar{M}, q)$ is finite, it satisfies the naive Ward identity(electric charge conservation):

$$
q_{\mu} \Pi^{5 R \mu \nu}(M, \bar{M}, q)=0
$$

On the other hand, the axial Ward identity is, formally:

$$
\begin{aligned}
& i\left(\Pi^{15 \mu \nu}+\Pi^{25 \mu \nu}\right) q_{\nu}=2 M \mathcal{A}(M, q)^{\mu} \\
& \quad=2 M(-i e)^{2} \int d p \operatorname{Tr}\left\{\left[\gamma^{\mu}+\frac{1}{2} n^{\mu}(\not h) m^{2}(n \cdot(p+q))^{-1}(n \cdot p)^{-1}\right] \frac{i\left(\not p+M-\frac{m^{2}}{2} \frac{h}{n \cdot p}\right)}{p^{2}-M^{2}-m^{2}+i \varepsilon} \gamma^{5} \frac{i\left((\not p+\not q)+M-\frac{m^{2}}{2} \frac{n}{n \cdot(p+q)}\right)}{(p+q)^{2}-M^{2}-m^{2}+i \varepsilon}\right\}
\end{aligned}
$$

if shift of the integration variable $p \rightarrow p+k$ is allowed.

Therefore the regularized amplitude satisfies:

$$
i \Pi^{5 R \mu \nu}(M, \bar{M}, q) q_{\nu}=2 M \mathcal{A}(M, q)^{\mu}-2 \bar{M} \mathcal{A}(\bar{M}, q)^{\mu} .
$$

Since the original amplitude is obtained formally as $\lim _{\bar{M} \rightarrow \infty}$, the axial anomaly is given by:

$$
B^{\mu}=\lim _{\bar{M} \rightarrow \infty}\left(-2 \bar{M} \mathcal{A}(\bar{M}, q)^{\mu}\right) .
$$

Now, we compute (5). First notice that after computing the trace, the integral is finite. A typical term containing the vector $n^{\mu}$ is of the form:

$$
C^{\mu}=2 M^{2} m^{2}(-i e)^{2} \varepsilon^{\mu \alpha} n_{\alpha} \int d p \frac{1}{p^{2}-M^{2}-m^{2}+i \varepsilon} \frac{1}{(p+q)^{2}-M^{2}-m^{2}+i \varepsilon} \frac{1}{n \cdot p} .
$$

Now we recall an important property of ML prescription. It preserves naive power counting. According to this, $C^{\mu} \sim M^{-1}$ for large $M$.

Following the same argument, we can easily check that all terms containing $n^{\mu}$ vanish when $M \rightarrow \infty$.

It remains the Lorentz invariant term:

$$
i \Pi^{5 \mu \nu}(q) q_{\nu}=\lim _{\bar{M} \rightarrow \infty}-4 e^{2} \bar{M}^{2} \varepsilon^{\alpha \mu} q_{\alpha} \int d p \frac{1}{p^{2}-\bar{M}^{2}-m^{2}+i \varepsilon} \frac{1}{(p+q)^{2}-\bar{M}^{2}-m^{2}+i \varepsilon}=-i \frac{e^{2}}{\pi} \varepsilon^{\alpha \mu} q_{\alpha}
$$




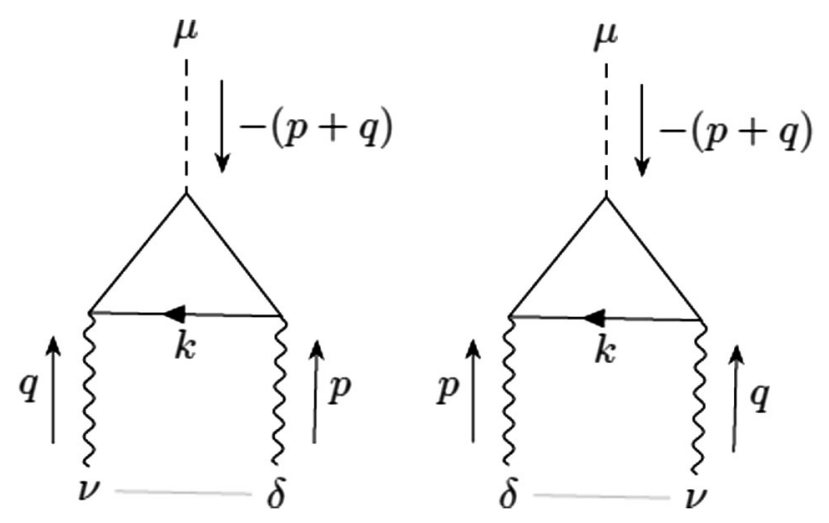

FIG. 3. $\Pi^{15 \mu \nu \delta}$.

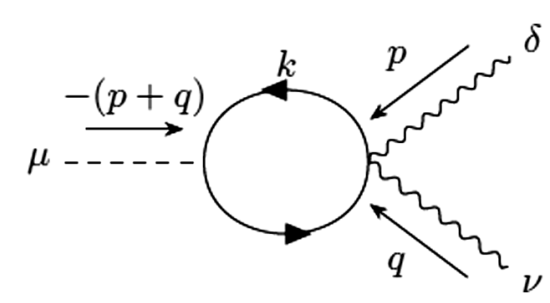

FIG. 4. $\Pi^{25 \mu \nu \delta}$.

$$
q_{\nu}\left\langle j^{5 \nu}\right\rangle=\frac{1}{-i e} i \Pi^{5 \mu \nu}(q) A_{\mu} q_{\nu}=\frac{e}{\pi} \varepsilon^{\alpha \mu} q_{\alpha} A_{\mu}
$$

Equation (7) is the standard Lorentz invariant result [26].

We want to comment on a previous computation of the anomaly in [20]. There and here, the vector current is conserved, but a different axial anomaly is obtained. This

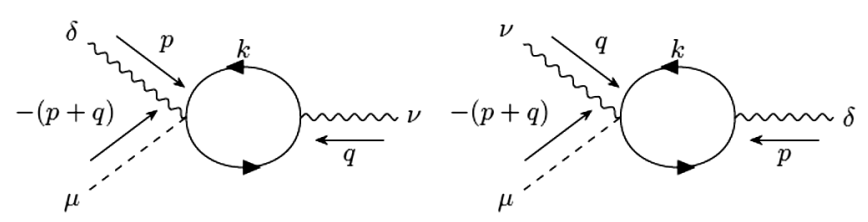

FIG. 5. $\Pi^{35 \mu \nu \delta}$.

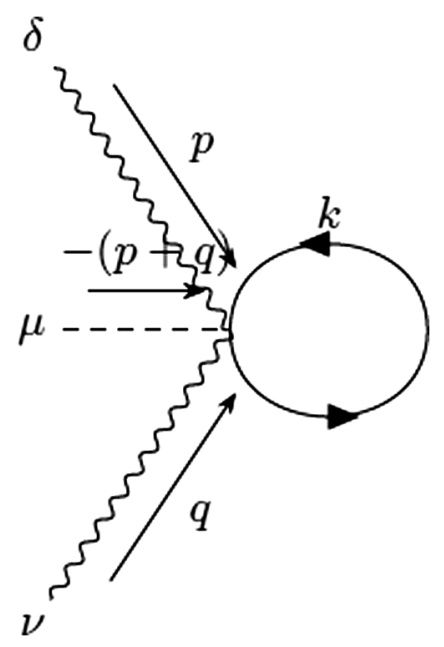

FIG. 6. $\Pi^{45 \mu \nu \delta}$.

difference may be a result of different normalization conditions [27] or the extra freedom we have when Lorentz symmetry is broken [28].

It is clear though that the procedure used in [20] does not respect naive power counting of the loop integrals.

\section{FOUR DIMENSIONAL AXIAL ANOMALY}

We compute:

$$
\int d^{4} x e^{-i r x}\left\langle p, q\left|j^{\mu 5}(x)\right| 0\right\rangle=(2 \pi)^{4} \delta(-r+p+q) \varepsilon_{\nu}^{*}(q) \varepsilon_{\delta}^{*}(p) i \Pi^{5 \mu \nu \delta}
$$

There are four graphs that contribute to the axial anomaly in four dimensions (Figs. 3-6). Notice that in [22] Figs. 5 and 6 are missing. They are fundamental to satisfy the Ward identity for the vector current(charge conservation) as well as the right computation of the axial anomaly.

$$
\begin{aligned}
i \Pi^{15 \mu \nu \delta}= & -(-i e)^{2} \int d k \operatorname{Tr}\left\{\left[\gamma^{\mu}+\frac{1}{2} n^{\mu}(\not h) m^{2}(n \cdot(k+q))^{-1}(n \cdot(k-p))^{-1}\right] \gamma^{5} \frac{i\left((\not k+\not k)+M-\frac{m^{2}}{2} \frac{\hbar}{n \cdot(k+q)}\right)}{(k+q)^{2}-M^{2}-m^{2}+i \varepsilon}\right. \\
& \times\left[\gamma^{\nu}+\frac{1}{2} n^{\nu}(\not h) m^{2}(n \cdot(k+q))^{-1}(n \cdot k)^{-1}\right] \frac{i\left(\not k+M-\frac{m^{2}}{2} \frac{\not}{n \cdot k}\right)}{k^{2}-M^{2}-m^{2}+i \varepsilon} \\
& \left.\times\left[\gamma^{\delta}+\frac{1}{2} n^{\delta}(\not h) m^{2}(n \cdot(k-p))^{-1}(n \cdot k)^{-1}\right] \frac{i\left(\not k-\not p+M-\frac{m^{2}}{2} \frac{\hbar}{n \cdot(k-p)}\right)}{(k-p)^{2}-M^{2}-m^{2}+i \varepsilon}\right\}+(p, \delta) \rightarrow(q, \nu)
\end{aligned}
$$




$$
\begin{aligned}
i \Pi^{25 \mu \nu \delta}= & (-1)(i e)^{2} n^{\delta} n^{\nu} i \int d k(n \cdot k)^{-1}(n \cdot(k-p-q))^{-1}\left[(n \cdot(k-q))^{-1}+(n \cdot(k-p))^{-1}\right] \\
& \times \operatorname{Tr}\left\{\frac{1}{2} \not h m^{2} \frac{i\left(\not k-\not p-\not 1+M-\frac{m^{2}}{2} \frac{\not h}{n \cdot(k-p-q)}\right)}{(k-p-q)^{2}-M^{2}-m^{2}+i \varepsilon}\left[\gamma^{\mu}+\frac{1}{2} n^{\mu}(\not h) m^{2}(n \cdot k)^{-1}(n \cdot(k-p-q))^{-1}\right] \gamma^{5} \frac{i\left(\not k+M-\frac{m^{2}}{2} \frac{\not}{n \cdot k}\right)}{k^{2}-M^{2}-m^{2}+i \varepsilon}\right\}
\end{aligned}
$$

$$
\begin{aligned}
i \Pi^{35 \mu \nu \delta}= & (-1)(i e)^{2} n^{\delta} n^{\mu} i \int d k(n \cdot k)^{-1}(n \cdot(k-q))^{-1}\left[(n \cdot(k-q-p))^{-1}+(n \cdot(k+p))^{-1}\right] \\
& \times \operatorname{Tr}\left\{\frac{1}{2} \not h m^{2} \gamma^{5} \frac{i\left(\not k+M-\frac{m^{2}}{2} \frac{\hbar}{n \cdot k}\right)}{k^{2}-M^{2}-m^{2}+i \varepsilon}\left[\gamma^{\nu}+\frac{1}{2} n^{\nu}(\not h) m^{2}(n \cdot k)^{-1}(n \cdot(k-q))^{-1}\right] \frac{i\left(\not k-\not 1+M-\frac{m^{2}}{2} \frac{\hbar}{n \cdot(k-q)}\right)}{(k-q)^{2}-M^{2}-m^{2}+i \varepsilon}\right\} \\
& +(p, \delta) \rightarrow(q, \nu)
\end{aligned}
$$

$$
\begin{aligned}
i \Pi^{45 \mu \nu \delta}= & (-1)(i e)^{2} n^{\nu} n^{\mu} n^{\delta} i \int d k\left\{\frac { 1 } { n \cdot k } \frac { 1 } { n \cdot k } \left[\frac{1}{n \cdot(k+p+q)} \frac{1}{n \cdot(k+p)}+\frac{1}{n \cdot(k+p+q)} \frac{1}{n \cdot(k+q)}\right.\right. \\
& \left.\left.+\frac{1}{n \cdot(k-p)} \frac{1}{n \cdot(k-p-q)}+\frac{1}{n \cdot(k-q)} \frac{1}{n \cdot(k+p)}+\frac{1}{n \cdot(k-p)} \frac{1}{n \cdot(k+q)}+\frac{1}{n \cdot(k-q)} \frac{1}{n \cdot(k-p-q)}\right]\right\} \\
& \times \operatorname{Tr}\left\{\frac{1}{2} \not h m^{2} \gamma^{5}[i] \frac{i\left(\not k+M-\frac{m^{2}}{2} \frac{n}{n \cdot(k)}\right)}{(k)^{2}-M^{2}-m^{2}+i \varepsilon}\right\}
\end{aligned}
$$

Notice that $\Pi^{25 \mu \nu \delta}, \Pi^{35 \mu \nu \delta}, \Pi^{45 \mu \nu \delta}$ are ultraviolet finite. Only $\Pi^{15 \mu \nu \delta}$ is linearly divergent as in the Lorentz invariant electrodynamics.

To compute the axial anomaly we will use Pauli-Villars regularization and Mandelstam-Leibbrandt prescription to treat infrared divergences. We will follow Ref. [27].

It is easy to check that formally:

$$
\left(\Pi^{15 \mu \nu \delta}+\Pi^{25 \mu \nu \delta}+\Pi^{35 \mu \nu \delta}+\Pi^{45 \mu \nu \delta}\right) p_{\delta}=0
$$

if shift of the integration variable $k \rightarrow k+Q$ is allowed. Here $Q$ is a constant vector. ${ }^{1}$

Introduce a Pauli-Villars particle of mass $\bar{M}$ and define the regularized amplitude:

$$
\Pi^{5 R \mu \nu \delta}(M, \bar{M}, p, q)=\left(\Pi^{15 \mu \nu \delta}+\Pi^{25 \mu \nu \delta}+\Pi^{35 \mu \nu \delta}+\Pi^{45 \mu \nu \delta}\right)(M, p, q)-\left(\Pi^{15 \mu \nu \delta}+\Pi^{25 \mu \nu \delta}+\Pi^{35 \mu \nu \delta}+\Pi^{45 \mu \nu \delta}\right)(\bar{M}, p, q)
$$

Since $\Pi^{5 R \mu \nu \delta}(M, \bar{M}, p, q)$ is finite, it satisfies the naive Ward identity(electric charge conservation):

$$
\Pi^{5 R \mu \nu \delta}(M, \bar{M}, p, q) p_{\delta}=0
$$

Besides, the axial Ward identity formally is, if shift of the integration variable $k \rightarrow k+Q$ is allowed:

$$
\begin{aligned}
-(p+q)_{\mu} i\left(\Pi^{15 \mu \nu \delta}+\Pi^{25 \mu \nu \delta}+\Pi^{35 \mu \nu \delta}+\Pi^{45 \mu \nu \delta}\right)=2 M \mathcal{A}(M, p, q)^{\nu \delta} \\
=-2 M(-i e)^{2} \int d k\left\{\operatorname { T r } \left\{\gamma^{5} \frac{i\left((\not k+\not p+\not 1)+M-\frac{m^{2}}{2} \frac{\hbar}{n \cdot(k+p+q)}\right)}{(k+p+q)^{2}-M^{2}-m^{2}+i \varepsilon}\left[\gamma^{\nu}+\frac{1}{2} n^{\nu}(\not h) m^{2}(n \cdot(k+p+q))^{-1}(n \cdot(k+p))^{-1}\right]\right.\right. \\
\left.\left.\quad \times \frac{i\left(\not k+\not p+M-\frac{m^{2}}{2} \frac{\hbar}{n \cdot(k+p)}\right)}{(k+p)^{2}-M^{2}-m^{2}+i \varepsilon}\left[\gamma^{\delta}+\frac{1}{2} n^{\delta}(\not h) m^{2}(n \cdot(k+p))^{-1}(n \cdot k)^{-1}\right] \frac{i\left(\not k+M-\frac{m^{2}}{2} \frac{\not h}{n \cdot(k)}\right)}{(k)^{2}-M^{2}-m^{2}+i \varepsilon}\right\}+(p, \delta) \rightarrow(q, \nu)\right\}
\end{aligned}
$$

\footnotetext{
${ }^{1}$ This is true if we use DR as in Secs. V and VI.
} 


$$
\begin{aligned}
& (-2 M)(i e)^{2} n^{\delta} n^{\nu} i \int d k(n \cdot k)^{-1}(n \cdot(k-p-q))^{-1}\left[(n \cdot(k-q))^{-1}+(n \cdot(k-p))^{-1}\right] \\
& \quad \times \operatorname{Tr}\left[\frac{1}{2} \not h m^{2} \frac{i\left(\not k-\not p-\not k+M-\frac{m^{2}}{2} \frac{n}{n \cdot(k-p-q)}\right)}{(k-p-q)^{2}-M^{2}-m^{2}+i \varepsilon} \gamma^{5} \frac{i\left(\not k+M-\frac{m^{2}}{2} \frac{h}{n \cdot k}\right)}{k^{2}-M^{2}-m^{2}+i \varepsilon}\right]
\end{aligned}
$$

The term (13) is convergent and has zero trace in four dimensions. So it vanishes.

Therefore the regularized amplitude satisfies:

$$
-(p+q)_{\mu} i \Pi^{5 R \mu \nu \delta}(M, \bar{M}, p, q)=2 M \mathcal{A}(M, p, q)^{\nu \delta}-2 \bar{M} \mathcal{A}(\bar{M}, p, q)^{\nu \delta}
$$

Since the original amplitude is obtained formally as $\lim _{\bar{M} \rightarrow \infty}$, the axial anomaly is given by:

$$
A^{\nu \delta}=\lim _{\bar{M} \rightarrow \infty}\left(-2 \bar{M} \mathcal{A}(\bar{M}, p, q)^{\nu \delta}\right)
$$

After computing the trace, we use ML prescription to regulate the infrared divergences. $\mathcal{A}(M, p, q)^{\nu \delta}$ is ultraviolet finite

A nice property of ML prescription is that preserve naive power counting. Using this property, we can easily show that all terms containing $n^{\mu}$ in $\mathcal{A}(M, p, q)^{\nu \delta}$ are smaller than $M^{-2}$ for large $M$, so they do not contribute to the axial anomaly.

$$
\mathcal{A}^{\nu \delta}=\lim _{\bar{M} \rightarrow \infty} 8 \bar{M}^{2} \varepsilon^{\nu \delta \alpha \beta} p_{\alpha} q_{\beta}(-i e)^{2} \int d^{4} k \frac{1}{(k+p)^{2}-m^{2}-\bar{M}^{2}} \frac{1}{k^{2}-m^{2}-\bar{M}^{2}} \frac{1}{(k+p)^{2}-m^{2}-\bar{M}^{2}}
$$

That is:

$$
\mathcal{A}^{\nu \delta}=-(i e)^{2} \frac{i}{2 \pi^{2}} \varepsilon^{\nu \delta \beta \mu} p_{\beta} q_{\mu}
$$

This is the standard result [26,27].

We see that Pauli-Villars regularization of ultraviolet divergences and Mandelstam-Leibbrandt regularization of infrared divergences preserve the Ward identity for the vector current (electric charge conservation) as well as the standard anomaly for the axial current, without modification from VSR terms.

\section{TWO DIMENSIONAL AXIAL ANOMALY IN DIMENSIONAL REGULARIZATION}

To treat $\gamma^{5}$ we follow the prescription of [23]. That is, in any number of dimensions

$$
\begin{gathered}
\gamma^{5}=i \gamma^{0} \gamma^{1} \\
\left\{\gamma^{5}, \gamma^{\mu}\right\}=0 . \mu=0,1 ; \quad\left[\gamma^{5}, \gamma^{\mu}\right]=0, \quad \mu=2,3 \ldots, d \\
q_{\mu}, n^{\mu} \text { are two dimensional vectors. } \quad p_{\mu} \text { is } d-\text { dimensional } \\
i \Pi^{15 \mu \nu} q_{\nu}=-(-i e)^{2} \int d p \operatorname{Tr}\left\{\left[\gamma^{\mu}+\frac{1}{2} n^{\mu}(\not h) m^{2}(n \cdot(p+q))^{-1}(n \cdot p)^{-1}\right] \frac{i\left(\not p+M-\frac{m^{2}}{2} \frac{\not h}{n \cdot p}\right)}{p^{2}-M^{2}-m^{2}+i \varepsilon}\right. \\
\left.\times\left(\not q+\frac{1}{2} n \cdot q(\not h) m^{2}(n \cdot(p+q))^{-1}(n \cdot p)^{-1}\right) \gamma^{5} \frac{i\left((\not p+\not 1)+M-\frac{m^{2}}{2} \frac{\not n}{n \cdot(p+q)}\right)}{(p+q)^{2}-M^{2}-m^{2}+i \varepsilon}\right\}
\end{gathered}
$$

Write

$$
\not p=\not p_{1}+\not \not_{2} ; 1 \text { lives in two dimensions, } 2 \text { lives in } d-2 \text { dimensions }
$$

Now we use the identity:

$$
\left[\not q+\frac{1}{2} n \cdot q(\not h) m^{2}(n \cdot(p+q))^{-1}(n \cdot p)^{-1}\right]=\left[\not p+\not q-\frac{1}{2} \not h m^{2}(n \cdot(p+q))^{-1}-M-\left(\not p-\frac{m^{2} \not h}{2 n \cdot p}-M\right)\right]
$$




$$
\begin{aligned}
& \Pi^{15 \mu \nu} q_{\nu}=-(-i e)^{2} \int d p \operatorname{Tr}\left\{-\left[\gamma^{\mu}+\frac{1}{2} n^{\mu}(\not h) m^{2}(n \cdot(p+q))^{-1}(n \cdot p)^{-1}\right] \gamma_{5} \frac{i\left((\not p+\not q)+M-\frac{m^{2}}{2} \frac{\not h}{n \cdot(p+q)}\right)}{(p+q)^{2}-M^{2}-m^{2}+i \varepsilon}\right. \\
& +\left[\gamma^{\mu}+\frac{1}{2} n^{\mu}(\not h) m^{2}(n \cdot(p+q))^{-1}(n \cdot p)^{-1}\right] \frac{i\left(\not p+M-\frac{m^{2}}{2} \frac{\not}{n \cdot p}\right)}{p^{2}-M^{2}-m^{2}+i \varepsilon} \gamma_{5}(-) \\
& \left.\times\left[\left(\not \not p-\not \not p 2+\not q-\frac{1}{2} \not h m^{2}(n \cdot(p+q))^{-1}-M\right)+2 M\right] \frac{i\left((\not p+\not q)+M-\frac{m^{2}}{2} \frac{n}{n \cdot(p+q)}\right)}{(p+q)^{2}-M^{2}-m^{2}+i \varepsilon}\right\} \\
& =-(-i e)^{2} \int d p \operatorname{Tr}\left\{\gamma^{5}\left[\gamma^{\mu}+\frac{1}{2} n^{\mu}(\not h) m^{2}(n \cdot(p+q))^{-1}(n \cdot p)^{-1}\right] \frac{i\left((\not p+\not q)+M-\frac{m^{2}}{2} \frac{\hbar}{n \cdot(p+q)}\right)}{(p+q)^{2}-M^{2}-m^{2}+i \varepsilon}-\right. \\
& \left.\gamma^{5}\left[\gamma^{\mu}+\frac{1}{2} n^{\mu}(\not h) m^{2}(n \cdot(p+q))^{-1}(n \cdot p)^{-1}\right] \frac{i\left(\not p+M-\frac{m^{2}}{2} \frac{\hbar}{n \cdot p}\right)}{p^{2}-M^{2}-m^{2}+i \varepsilon}\right\} \\
& +2 M(-i e)^{2} \int d p \operatorname{Tr}\left\{\left[\gamma^{\mu}+\frac{1}{2} n^{\mu}(\not h) m^{2}(n \cdot(p+q))^{-1}(n \cdot p)^{-1}\right] \frac{i\left(\not p+M-\frac{m^{2}}{2} \frac{\not}{n \cdot p}\right)}{p^{2}-M^{2}-m^{2}+i \varepsilon} \gamma_{5} \frac{i\left((\not p+\not q)+M-\frac{m^{2}}{2} \frac{\hbar}{n \cdot(p+q)}\right)}{(p+q)^{2}-M^{2}-m^{2}+i \varepsilon}\right\} \\
& -(-i e)^{2} \int d p \operatorname{Tr}\left\{\left[\gamma^{\mu}+\frac{1}{2} n^{\mu}(\not h) m^{2}(n \cdot(p+q))^{-1}(n \cdot p)^{-1}\right] \frac{i\left(\not p+M-\frac{m^{2}}{2} \frac{\not}{n \cdot p}\right)}{p^{2}-M^{2}-m^{2}+i \varepsilon} \gamma^{5} 2 \not p_{2} \frac{i\left((\not p+\not q)+M-\frac{m^{2}}{2} \frac{\hbar}{n \cdot(p+q)}\right)}{(p+q)^{2}-M^{2}-m^{2}+i \varepsilon}\right\} \text {. }
\end{aligned}
$$

In dimensional regularization we can shift variable $p \rightarrow p-q$ in the term (16). Then the addition of terms (16) and (17)) is cancelled by the contribution of Fig. 2 .

The anomaly is

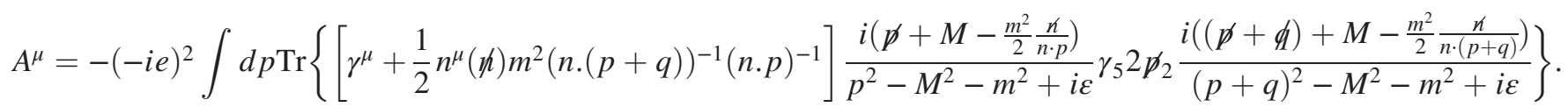

That is

$$
A^{\mu}=4(-i e)^{2} \int d p \frac{\left[-p_{2}^{2} \varepsilon^{\mu \nu} q_{\nu}-p_{2}^{2} \frac{1}{2} n^{\mu} m^{2}(n \cdot(p+q))^{-1}(n \cdot p)^{-1} \varepsilon^{\alpha \beta} n_{\alpha} q_{\beta}\right]}{\left(p^{2}-M^{2}-m^{2}+i \varepsilon\right)\left((p+q)^{2}-M^{2}-m^{2}+i \varepsilon\right)}
$$

$p_{2}^{2} \sim(d-2) p^{2}$ when $d \rightarrow 2$. The VSR part of the integral is convergent, using ML prescription, so it is zero, when we take $d=2$.

So only the Lorentz invariant part of the integral contributes to the anomaly.

$$
A^{\mu}=4 e^{2} \varepsilon^{\mu \nu} q_{\nu} \int d p \frac{p_{2}^{2}}{p^{2}-M^{2}-m^{2}+i \varepsilon} \frac{}{(p+q)^{2}-M^{2}-m^{2}+i \varepsilon}=e^{2} \varepsilon^{\mu \nu} q_{\nu} \frac{i}{\pi} .
$$

That is

$$
q_{\mu}\left\langle j^{5 \mu}(q)\right\rangle=\frac{e^{2}}{-i e} \varepsilon^{\mu \nu} q_{\nu} \frac{i}{\pi} A_{\mu}=-\frac{e}{\pi} \varepsilon^{\mu \nu} q_{\nu} A_{\mu}=\frac{e}{\pi} \varepsilon^{\nu \mu} q_{\nu} A_{\mu}
$$

which is the standard result [26].

In Appendix B we study the vector Ward identity. If we use dimensional regularization there, then shifting the integration variable $p->p+Q$ is allowed. So the naive Ward identity for the vector current is satisfied without anomaly. 


\section{4D AXIAL ANOMALY. DIMENSIONAL REGULARIZATION}

In this section we compute the axial anomaly using dimensional regularization. The contribution of Fig. 3 is

$$
\begin{aligned}
-(p+q)_{\mu} i \Pi^{15 \mu \nu \delta}= & -(-i e)^{2} \int d k \operatorname{Tr}\left\{\left[-(\not p+\not q)-\frac{1}{2}(p+q) \cdot n(\not h) m^{2}(n \cdot(k+q))^{-1}(n \cdot(k-p))^{-1}\right] \gamma^{5}\right. \\
& \times \frac{i\left((\not k+\not q)+M-\frac{m^{2}}{2} \frac{\not h}{n \cdot(k+q)}\right)}{(k+q)^{2}-M^{2}-m^{2}+i \varepsilon}\left[\gamma^{\nu}+\frac{1}{2} n^{\nu}(\not h) m^{2}(n \cdot(k+q))^{-1}(n \cdot k)^{-1}\right] \frac{i\left(\not k+M-\frac{m^{2}}{2} \frac{\hbar}{n \cdot k}\right)}{k^{2}-M^{2}-m^{2}+i \varepsilon} \\
& \left.\times\left[\gamma^{\delta}+\frac{1}{2} n^{\delta}(\not h) m^{2}(n \cdot(k-p))^{-1}(n \cdot k)^{-1}\right] \frac{i\left(\not k-\not p+M-\frac{m^{2}}{2} \frac{\hbar}{n \cdot(k-p)}\right)}{(k-p)^{2}-M^{2}-m^{2}+i \varepsilon}\right\} .
\end{aligned}
$$

Write $\not h=\not K K_{1}+\not K_{2}$

$$
\left(\not l_{1}+\not k_{2}+\not p+\not h+M\right) \gamma_{5}=-\gamma_{5}\left(\not \not_{1}+\not \not_{2}+\not p+\not q+M\right)+2 \gamma_{5} \not \not_{2}+2 M \gamma_{5} .
$$

That is the anomaly is

$$
\begin{aligned}
\Gamma^{5 \nu \delta}(p, q)= & 2(-i e)^{2} \int d k \operatorname{Tr}\left\{\gamma^{5} \not k_{2} \frac{i\left((\not k+\not p+\not q)+M-\frac{m^{2}}{2} \frac{\hbar k}{n \cdot(k+p+q)}\right)}{(k+p+q)^{2}-M^{2}-m^{2}+i \varepsilon}\left[\gamma^{\nu}+\frac{1}{2} n^{\nu}(\not h) m^{2}(n \cdot(k+p+q))^{-1}(n \cdot(k+p))^{-1}\right]\right. \\
& \left.\times \frac{i\left(\not k+\not h+M-\frac{m^{2}}{2} \frac{n}{n \cdot(k+p)}\right)}{(k+p)^{2}-M^{2}-m^{2}+i \varepsilon}\left[\gamma^{\delta}+\frac{1}{2} n^{\delta}(\not h) m^{2}(n \cdot(k+p))^{-1}(n \cdot k)^{-1}\right] \frac{i\left(\not k+M-\frac{m^{2}}{2} \frac{h}{n \cdot(k)}\right)}{(k)^{2}-M^{2}-m^{2}+i \varepsilon}\right\}
\end{aligned}
$$

To compute the trace, we notice that there must be an even number of $\not k_{2}$ otherwise the trace vanishes. Assume there are four $\not k_{2}$

$$
\operatorname{Tr}\left\{\gamma^{5} \not k_{2} \not k_{2} \gamma^{\nu} \not k_{2} \gamma^{\delta} \not k_{2}\right\}=\left(k_{2}^{2}\right)^{2} \operatorname{Tr}\left\{\gamma^{5} \gamma^{\nu} \gamma^{\delta}\right\}=0
$$

That is, only two $\not k_{2}$ contribute to the trace.

The trace can be written as $\operatorname{Tr}=k_{2}^{2} S$

But $k_{2}^{2} S \sim(d-4) k^{2} S$. So if $k^{2} S$ is convergent in $d=4$ the contribution of this $S$ vanishes. If we use ML prescription to regularize the infrared divergences we can show that $k^{2} S$ is convergent in $d=4$ for all VSR $S$ 's, since ML preserves naive power counting. Therefore only Lorentz invariant terms contribute to the anomaly.

Finally the anomaly is

$$
\begin{aligned}
\Gamma^{5 \nu \delta}(p, q) & =2(-i e)^{2} i^{3} \int d k k_{2}^{2} \frac{\operatorname{Tr}\left\{\gamma_{5}(\not h) \gamma_{\nu}(\not p) \gamma_{\delta}\right\}}{(k+p+q)^{2}-M^{2}-m^{2}+i \varepsilon} \frac{1}{(k+p)^{2}-M^{2}-m^{2}+i \varepsilon} \frac{1}{(k)^{2}-M^{2}-m^{2}+i \varepsilon} \\
& =-i \frac{e^{2}}{2 \pi^{2}} \varepsilon^{\nu \delta \alpha \mu} p_{\alpha} q_{\mu}
\end{aligned}
$$

Therefore

$$
\left\langle p, q\left|\partial_{\mu} j^{5 \mu}(0)\right| 0\right\rangle=-\frac{e^{2}}{2 \pi^{2}} \varepsilon^{\mu \nu \alpha \delta}\left(-i q_{\mu}\right) \varepsilon_{\nu}^{*}(q)\left(-i p_{\alpha}\right) \varepsilon_{\delta}^{*}(p)
$$

which is the standard result [26].

Following the same reasoning as in Appendix B, we can study the vector Ward identity in four dimensions. If we use dimensional regularization there, then shifting the integration variable $k->k+Q$ is allowed. So the naive Ward identity for the vector current is satisfied without anomaly.

\section{PATH INTEGRAL DERIVATION OF THE AXIAL ANOMALY}

We use the approach of [29]. 
The generating functional in the presence of an external field $A_{\mu}$ is

$$
Z=\int \mathcal{D} \psi \mathcal{D} \bar{\psi} e^{i \int d^{4} x \bar{\psi} i \not \psi \psi}
$$

where the gauge invariant and $\operatorname{Sim}(2)$ invariant Dirac operator is

$$
\mathcal{D}=D+\frac{1}{2} \not m^{2}(n \cdot D)^{-1}, D_{\mu}=\partial_{\mu}-i e A_{\mu} .
$$

Introduce a basis of eigenvectors of $\mathcal{D}$

$$
i \mathcal{D} \phi_{m}=\lambda_{m} \phi_{m}, \hat{\phi}_{m}(i \mathcal{D})=-i \mathcal{D}{ }_{\mu} \hat{\phi}_{m} \gamma^{\mu}=\lambda_{m} \hat{\phi}_{m}
$$

For large $q$ and fixed $A_{\mu}$

$$
\phi_{m}(x) \sim \phi_{m}(q) e^{i q x}, \quad \lambda_{m}^{2} \sim q^{2},
$$

We can expand

$$
\psi(x)=\sum_{m} a_{m} \phi_{m}(x), \quad \bar{\psi}(x)=\sum_{m} \bar{a}_{m} \hat{\phi}_{m}(x) .
$$

The integration measure is defined by:

$$
\mathcal{D} \psi \mathcal{D} \bar{\psi}=\prod_{m} d a_{m} d \bar{a}_{m} .
$$

Under the change of variables:

$$
\psi^{\prime}(x)=\left(1+i \alpha(x) \gamma^{5}\right) \psi(x)
$$

we get:

$$
\mathcal{D} \psi^{\prime} \mathcal{D} \bar{\psi}^{\prime}=\mathcal{J}^{-2} \mathcal{D} \psi \mathcal{D} \bar{\psi}
$$

where the Jacobian $\mathcal{J}$ is given by:

$$
\log \mathcal{J}=i \int d^{4} x \alpha(x) \sum_{n} \phi_{n}^{\dagger}(x) \gamma^{5} \phi_{n}(x)
$$

To evaluate it we introduce a gauge invariant and $\operatorname{Sim}(2)$ invariant regularization:

$$
\begin{aligned}
\sum_{n} \phi_{n}^{\dagger}(x) \gamma^{5} \phi_{n}(x) & =\lim _{M \rightarrow \infty} \sum_{n} \phi_{n}^{\dagger}(x) \gamma^{5} \phi_{n}(x) e^{\frac{\lambda_{n}^{2}}{M^{2}}} \\
& =\lim _{M \rightarrow \infty}\left\langle x\left|\operatorname{Tr}\left\{\gamma^{5} e^{\frac{(i \not \mathcal{B})^{2}}{M^{2}}}\right\}\right| x\right\rangle
\end{aligned}
$$

Tr traces over Dirac indices.

We can write:

$$
(i \mathcal{D})^{2}=-\mathcal{D}_{\mu} \mathcal{D}_{\nu} g^{\mu \nu}-\frac{1}{2}\left[\mathcal{D}_{\mu}, \mathcal{D}_{\nu}\right] \sigma^{\mu \nu}, \quad \sigma^{\mu \nu}=\frac{i}{2}\left[\gamma^{\mu}, \gamma^{\nu}\right]
$$

Since we take $M \rightarrow \infty$, we look at the asymptotic part of the spectrum.

It is simpler to evaluate the commutator in the light cone gauge $n . A=0$

$$
\begin{aligned}
{\left[\mathcal{D}_{\mu}, \mathcal{D}_{\nu}\right] \phi } & \sim-i e F_{\mu \nu}(p) \phi(q) e^{i(p+q) x}-i e \frac{1}{2} m^{2} \frac{n \cdot p}{n \cdot q(n \cdot p+n \cdot q)} i \phi(q) e^{i(p+q) x}\left(n_{\mu} A_{\nu}(p)-n_{\nu} A_{\mu}(p)\right) \\
& \sim-i e F_{\mu \nu}(p) \phi(q) e^{i(p+q) x} \text { for large } q \\
(i \mathcal{D})^{2} & =-\mathcal{D}_{\mu} \mathcal{D}_{\nu} g^{\mu \nu}+\frac{e}{2} F_{\mu \nu} \sigma^{\mu \nu} \lim _{M \rightarrow \infty}\left\langle x\left|\operatorname{Tr}\left\{\gamma^{5} e^{\frac{\left(i \mathcal{D}^{\mathcal{X}}\right)}{M^{2}}}\right\}\right| x\right\rangle \\
& =\lim _{M \rightarrow \infty} \operatorname{Tr}\left\{\frac{1}{2 !} \gamma^{5}\left(\frac{e}{2 M^{2}} F_{\mu \nu} \sigma^{\mu \nu}\right)^{2}\right\}\left\langle x\left|e^{-\frac{\partial^{2}}{M^{2}}}\right| x\right\rangle \\
\left\langle x\left|e^{-\frac{\partial^{2}}{M^{2}}}\right| x\right\rangle & =\lim _{x \rightarrow y} \int \frac{d^{4} k}{(2 \pi)^{4}} e^{-i k(x-y)} e^{\frac{k^{2}}{M^{2}}} \\
& =i \int \frac{d^{4} k_{E}}{(2 \pi)^{4}} e^{-\frac{k_{E}^{2}}{M^{2}}}=i \frac{M^{4}}{16 \pi^{2}}
\end{aligned}
$$

Then:

$$
\mathcal{J}=\exp \left(-i \int d^{4} x \alpha(x) \frac{e^{2}}{16 \pi^{2}} \varepsilon^{\alpha \beta \mu \nu} F_{\alpha \beta}(x) F_{\mu \nu}(x)\right) .
$$

$$
\lim _{M \rightarrow \infty}\left\langle x\left|\operatorname{Tr}\left\{\gamma^{5} e^{\frac{\left(i D^{\chi^{\gamma}}\right)}{M^{2}}}\right\}\right| x\right\rangle=-\frac{e^{2}}{32 \pi^{2}} \varepsilon^{\alpha \beta \mu \nu} F_{\alpha \beta}(x) F_{\mu \nu}(x) .
$$

That is:

Then the Adler-Bell-Jackiw anomaly follows.

Notice that we could get this result assuming that the infrared regulator of $\frac{1}{n \cdot q}$ preserves scaling (naive power 
counting). To guarantee this property we work with the ML prescription, as in the perturbative approach.

\section{CONCLUSIONS}

We have examined the appearance of axial anomalies in VSR electrodynamics, using Pauli-Villars and dimensional regularization of ultraviolet divergences and MandelstamLeibbrandt regularization of infrared divergences.

Given that ML preserves naive power counting in loop integrals, we have shown that the usual form for the anomaly of the axial current appears, without corrections from VSR terms. No anomaly is present in the vector current conservation. This computation is at variance from a previous result for the axial anomaly in two dimensions [20], where corrections from VSR terms were found. This difference could be due to different normalization conditions for the anomaly term[27] or some extra freedom that occurs when Lorentz invariance is violated [28]. In any case, our result implies that the procedure of [20] destroys the naive power counting of loop integrals.

The anomaly is produced because the loop integral is ultraviolet (UV) divergent, so a regulator must be introduced. To fix the renormalized quantities we must impose normalization conditions (Please see [27], chapter 13.1). These normalization conditions reflects the symmetries to be satisfied by the model. Different regulators may produce different normalization conditions and therefore the anomaly could appear in the vector current, axial vector current or in a combination of both (This is important for a chiral theory like the SM). See for example [30] where a regulator is able to interpolate between different forms of the anomaly.

In this paper we are discussing a theory that has infrared (IR) divergences as well. So a new ambiguity in the value of the loop integral appears. The result depends on the IR regulator we choose. Beside the normalization conditions on the renormalized quantities are different, because we have a new fixed vector $n_{\mu}$ and nonlocal terms are allowed.

The results contained in [20] and in this paper correspond to different normalization conditions. In this sense, both results are right. But naive power counting of loop integrals is such an important tool in quantum field theory that the normalization conditions of the present paper should be preferred.

In four dimension we find a completely different result compared to [22]. There they claim that the conservation for the vector current has an anomaly and VSR corrections should appear in the anomaly of the axial current. We notice also that Figs. 5 and 6 are lacking in the computation of both anomalies in [22]. Figures 5 and 6 are crucial to satisfy the Ward identity for the vector current as a procedure in $4 \mathrm{~d}$ similar to the one explained in Appendix B shows.

In Appendix $\mathrm{C}$ we derived the Ward identity for the product of two vectors and one axial vector current in VSR. The nonlocality of the model introduces new contact terms.
We study also the axial anomaly from the point of view of the path integral method. Again ML property of preserving scaling (naive power counting) permits us to show that the axial anomaly is the Lorentz invariant one, without corrections from VSR.

According to our results, using dimensional (or PV) regularization of UV divergences and ML regularization of IR divergences, the VSRSM must be free from local chiral anomalies. We get the same anomalies as in the SM, so the usual mechanism of cancellation of anomalies within families of leptons and quarks should work.

Finally we want to recall that $M$ is not the mass of the particle. So if the fermion acquires a VSR mass $m$ even if $M=0$, the divergence of the axial current will contain the anomalous term only.

\section{ACKNOWLEDGMENTS}

The research of J.A. was partially supported by the Institute of Physics, Pontificia Universidad Católica de Chile.

\section{APPENDIX A: FEYNMAN RULES}

$$
\begin{aligned}
& V\left(p_{1}, p_{2}, p_{3}, q\right) \\
& =i(i e)^{3} \frac{m^{2}}{2} \pi n^{\alpha_{1}} n^{\alpha_{2}} n^{\alpha_{3}} \frac{1}{n \cdot\left(q+p_{1}+p_{2}+p_{3}\right)} \\
& \times\left(\frac{1}{n \cdot\left(q+p_{1}+p_{2}\right)} \frac{1}{n \cdot\left(q+p_{1}\right)}+\frac{1}{n \cdot\left(q+p_{1}+p_{2}\right)} \frac{1}{n \cdot\left(q+p_{2}\right)}\right. \\
& +\frac{1}{n \cdot\left(q+p_{3}+p_{2}\right)} \frac{1}{n \cdot\left(q+p_{3}\right)}+\frac{1}{n \cdot\left(q+p_{1}+p_{3}\right)} \frac{1}{n \cdot\left(q+p_{1}\right)} \\
& \left.+\frac{1}{n \cdot\left(q+p_{2}+p_{3}\right)} \frac{1}{n \cdot\left(q+p_{2}\right)}+\frac{1}{n \cdot\left(q+p_{3}+p_{1}\right)} \frac{1}{n \cdot\left(q+p_{3}\right)}\right) \gamma^{5} \\
& \stackrel{p}{\longrightarrow} i \frac{\not p+M^{2}-\frac{m^{2}}{2} \frac{\not d}{n \cdot p}}{p^{2}-M^{2}-m^{2}+i \varepsilon}
\end{aligned}
$$

FIG. 7. Electron propagator.

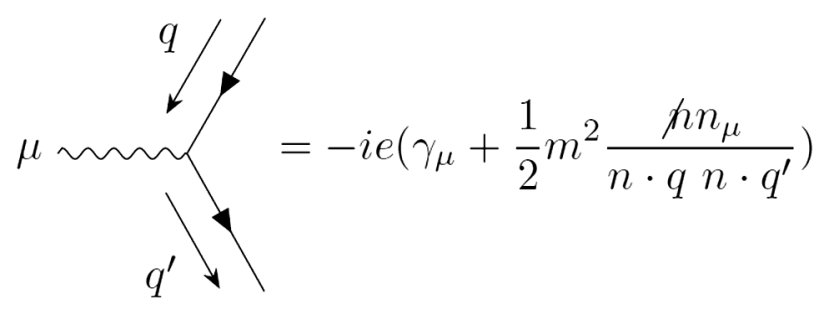

FIG. 8. $e-e-A_{\mu}$ vertex. 


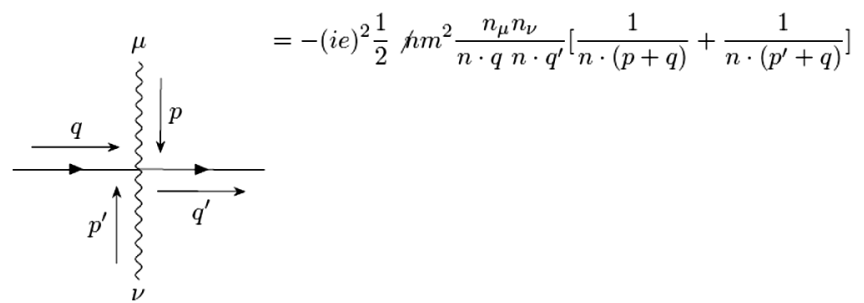

FIG. 9. $e-e-A_{\mu}-A_{\nu}$ vertex.

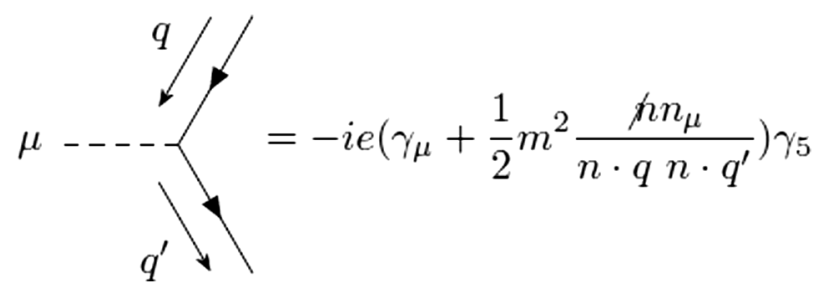

FIG. 10. axial-e-e vertex.

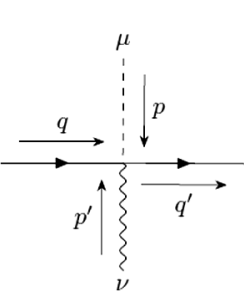

$=-(i e)^{2} \frac{1}{2} h m^{2} \frac{n_{\mu} n_{\nu}}{n \cdot q n \cdot q^{\prime}}\left[\frac{1}{n \cdot(p+q)}+\frac{1}{n \cdot\left(p^{\prime}+q\right)}\right] \gamma_{5}$

\section{APPENDIX B: FORMAL PROOF OF THE WARD IDENTITIES IN 2D}

In this Appendix we want to show in some detail how to obtain the Ward identities in $2 \mathrm{~d}$. In $4 \mathrm{~d}$ we have more graphs, but the procedure is essentially the same.

$$
\begin{aligned}
q_{\mu} \Pi^{15 \mu \nu}= & -(-i e)^{2} \int d p \operatorname{Tr}\left\{\left[\not q+\frac{1}{2} n \cdot q(\not h) m^{2}(n \cdot(p+q))^{-1}(n \cdot p)^{-1}\right] \frac{i\left(\not p+M-\frac{m^{2}}{2} \frac{\not h}{n \cdot p}\right)}{p^{2}-M^{2}-m^{2}+i \varepsilon}\right. \\
& \left.\times\left[\gamma^{\nu}+\frac{1}{2} n^{\nu}(\not h) m^{2}(n \cdot(p+q))^{-1}(n \cdot p)^{-1}\right] \gamma^{5} \frac{i\left((\not p+\not q)+M-\frac{m^{2}}{2} \frac{\not h}{n \cdot(p+q)}\right)}{(p+q)^{2}-M^{2}-m^{2}+i \varepsilon}\right\} .
\end{aligned}
$$

Now we use the identity:

$$
\left[\not q+\frac{1}{2} n \cdot q(\not h) m^{2}(n \cdot(p+q))^{-1}(n \cdot p)^{-1}\right]=\left[\not p+\not q-\frac{1}{2} \not h m^{2}(n \cdot(p+q))^{-1}-M-\left(\not p-\frac{m^{2} \not h}{2 n \cdot p}-M\right)\right]
$$

and the cyclic property of the trace to get:

$$
\begin{aligned}
q_{\mu} \Pi^{15 \mu \nu}= & (-i e)^{2} \int d p \operatorname{Tr}\left\{\frac{\left(\not p+M-\frac{m^{2}}{2} \frac{\not h}{n \cdot p}\right)}{p^{2}-M^{2}-m^{2}+i \varepsilon}\left[\gamma^{\nu}+\frac{1}{2} n^{\nu}(\not h) m^{2}(n \cdot(p+q))^{-1}(n \cdot p)^{-1}\right] \gamma^{5}\right. \\
& \left.-\left[\gamma^{\nu}+\frac{1}{2} n^{\nu}(\not h) m^{2}(n \cdot(p+q))^{-1}(n \cdot p)^{-1}\right] \gamma^{5} \frac{\left((\not p+\not q)+M-\frac{m^{2}}{2} \frac{n}{n \cdot(p+q)}\right)}{(p+q)^{2}-M^{2}-m^{2}+i \varepsilon}\right\} .
\end{aligned}
$$

Besides:

$$
q_{\mu} \Pi^{25 \mu \nu}=2(i e)^{2} n \cdot q n^{\nu} \int d p(n \cdot p)^{-1}\left[(n \cdot(q+p))^{-1}(n \cdot(-q+p))^{-1}\right] \operatorname{Tr} \frac{1}{2} \not h m^{2} \frac{\left(\not p+M-\frac{m^{2}}{2} \frac{\not}{n \cdot p}\right)}{p^{2}-M^{2}-m^{2}+i \varepsilon} \gamma^{5} .
$$


In the second term of (B2) shift $p \rightarrow p-q$ to get $^{2}$ :

$$
q_{\mu} \Pi^{15 \mu \nu}=-(-i e)^{2} \int d p \operatorname{Tr}\left\{\frac{1}{2} n^{\nu}(\not h) m^{2} \frac{\left(\not p+M-\frac{m^{2}}{2} \frac{\not h}{n \cdot p}\right)}{p^{2}-M^{2}-m^{2}+i \varepsilon} \gamma^{5}(n \cdot p)^{-1}(-2 n \cdot q)\left[(n \cdot(p+q))^{-1}(n \cdot(p-q))^{-1}\right]\right\} .
$$

That is $\Pi^{\mu \nu}=\Pi^{1 \mu \nu}+\Pi^{2 \mu \nu}$ is transverse.

The axial Ward identity is obtained in the same way.

\section{APPENDIX C: WARD IDENTITY FOR THE PRODUCT OF THREE CURRENTS IN VSR}

We follow [26] page 311.

The vector current is

$$
j^{\mu}=\bar{\psi} \gamma^{\mu} \psi+\frac{1}{2} m^{2} n^{\mu}\left((n \cdot \partial)^{-1} \bar{\psi}\right) \not h(n \cdot \partial)^{-1} \psi
$$

The axial vector current is

$$
j^{\mu 5}=\bar{\psi} \gamma^{\mu} \gamma^{5} \psi+\frac{1}{2} m^{2}\left(\frac{1}{n \cdot \partial} \bar{\psi}\right) \not h n^{\mu} \gamma^{5}\left(\frac{1}{n \cdot \partial} \psi\right) .
$$

Consider the path integral, where $S$ is the action (1) with $A_{\mu}=0$

$$
Z=\int \mathcal{D} \psi \mathcal{D} \bar{\psi} e^{i S} j^{\alpha 5}(y) j^{\beta}(z)
$$

Make the following local transformations

$$
\delta \psi(x)=i \alpha(x) \psi(x) ; \quad \delta \bar{\psi}(x)=-i \alpha(x) \bar{\psi}(x) .
$$

The integration measure is invariant under this transformation.

We get the following Ward identity:

$$
\left.\partial_{\mu}^{x}\left\langle 0\left|T\left(j^{\mu}(x) j^{\alpha 5}(y) j^{\beta}(z)\right)\right| 0\right\rangle-\langle 0| T \delta_{x} j^{\alpha 5}(y) j^{\beta}(z)\right)|0\rangle-\left\langle 0\left|T\left(j^{\alpha 5}(y) \delta_{x} j^{\beta}(z)\right)\right| 0\right\rangle=0
$$

where:

$$
\begin{aligned}
\delta_{x} j^{\mu}(y)= & \frac{1}{2} m^{2} n^{\mu}\left[\bar{\psi} \not h(n \cdot \partial)^{-1}(n \cdot \partial)^{-1} \psi \delta(x-y)+\bar{\psi} \not h(n \cdot \partial)^{-1} \psi(n \cdot \partial)^{-1} \delta(x-y)\right. \\
& -\left((n \cdot \partial)^{-1}(n \cdot \partial)^{-1} \bar{\psi}\right) \not h \psi \delta(x-y)-\left((n \cdot \partial)^{-1} \bar{\psi}\right) \not h \psi(n \cdot \partial)^{-1} \delta(x-y] \\
\delta_{x} j^{\mu 5}(y)= & \frac{1}{2} m^{2} n^{\mu}\left[\bar{\psi} \gamma^{5} \not h(n \cdot \partial)^{-1}(n \cdot \partial)^{-1} \psi \delta(x-y)+\bar{\psi} \not h \gamma^{5}(n \cdot \partial)^{-1} \psi(n \cdot \partial)^{-1} \delta(x-y)\right. \\
& -\left((n \cdot \partial)^{-1}(n \cdot \partial)^{-1} \bar{\psi}\right) \not h \gamma^{5} \psi \delta(x-y)-\left((n \cdot \partial)^{-1} \bar{\psi}\right) \not h \gamma^{5} \psi(n \cdot \partial)^{-1} \delta(x-y] .
\end{aligned}
$$

The nonlocality of the action and currents modify the Ward identity for the triangle graph. It is easy to check that the graphs in Fig. 3 satisfy (C1) if shifting of the loop integration variable is allowed.

Notice that (C1) is not given by the addition of the graphs in Fig. 1 of [22].

In a similar way we can derive the Ward identity for the divergence of the axial vector current.

\footnotetext{
${ }^{2}$ This is justified if we use DR as in Secs. V and VI.
} 
[1] The CMS Collaboration, Evidence for the direct decay of the $125 \mathrm{GeV}$ Higgs boson to fermions, Nat. Phys. 10, 557 (2014).

[2] P. Langacker, The Standard Model and Beyond (CRC Press, A Taylor and Francis Group, Boca Raton, 2010).

[3] R. Mohapatra, Unification and Supersymmetry: The Frontiers of Quark-Lepton Physics, 3rd ed. (Springer, New York, 2002).

[4] A. G. Cohen and S. L. Glashow, Very Special Relativity, Phys. Rev. Lett. 97, 021601 (2006).

[5] A. Cohen and S. Glashow, A Lorentz-violating origin of neutrino mass?, arXiv:hep-ph/0605036.

[6] A. G. Cohen and D. Z. Freedman, $\operatorname{SIM}(2)$ and SUSY, J. High Energy Phys. 07 (2007) 039.

[7] J. Vohanka, Gauge theory and $\operatorname{SIM}(2)$ superspace, Phys. Rev. D 85, 105009 (2012).

[8] G. Gibbons, J. Gomis, and C. Pope, General very special relativity is Finsler geometry, Phys. Rev. D 76, 081701 (2007).

[9] W. Muck, Very special relativity in curved space-times, Phys. Lett. B 670, 95 (2008).

[10] M. Sheikh-Jabbari and A. Tureanu, Realization of CohenGlashow Very Special Relativity on Noncommutative Space-Time, Phys. Rev. Lett. 101, 261601 (2008).

[11] S. Das, S. Ghosh, and S. Mignemi, Noncommutative spacetime in very special relativity, Phys. Lett. A 375, 3237 (2011).

[12] D. Ahluwalia and S. Horvath, Very special relativity as relativity of dark matter: The Elko connection, J. High Energy Phys. 11 (2010) 078.

[13] Z. Chang, M.-H. Li, X. Li, and S. Wang, Cosmological model with local symmetry of very special relativity and constraints on it from supernovae, Eur. Phys. J. C 73, 2459 (2013).

[14] J. Alfaro, P. González, and R. Ávila, Electroweak standard model with very special relativity, Phys. Rev. D 91, 105007 (2015); 91, 129904 (2015).

[15] S. Mandelstam, Light-cone superspace and the ultraviolet finiteness of the $N=4$ model, Nucl. Phys. B213, 149 (1983).
[16] G. Leibbrandt, Light-cone gauge in Yang-Mills theory, Phys. Rev. D 29, 1699 (1984).

[17] J. Alfaro, Mandelstam-Leibbrandt prescription, Phys. Rev. D 93, 065033 (2016); Erratum, Phys. Rev. D 94, 049901 (2016).

[18] J. Alfaro, A Sim(2) invariant dimensional regularization, Phys. Lett. B 772, 100 (2017).

[19] J. Alfaro, Feynman rules, ward identities and loop corrections in very special relativity standard model, Universe $\mathbf{5}$, 16 (2019).

[20] J. Alfaro and A. Soto, Schwinger model à la very special relativity, Phys. Lett. B 797, 134923 (2019).

[21] J. Alfaro and A. Soto, Photon mass in very special relativity, Phys. Rev. D 100, 055029 (2019).

[22] R. Bufalo, M. Ghasemkhani, and A. Soto, AdlerBell-Jackiw anomaly in VSR electrodynamics, arXiv:2011 .10649.

[23] G. t Hooft and M. J. G. Veltman, Regularization and renormalization of gauge fields, Nucl. Phys. B44, 189 (1972).

[24] S. Cheon, C. Lee, and S. J. Lee, SIM(2)-invariant modifications of electrodynamic theory, Phys. Lett. B 679, 73 (2009).

[25] J. Alfaro and V. O. Rivelles, Non-Abelian fields in very special relativity, Phys. Rev. D 88, 085023 (2013).

[26] M. E. Peskin and D. V. Schroeder, An Introduction to Quantum Field Theory (Addison-Wesley, Reading, MA, 1995), Chap. 19.1. The convention is $\epsilon^{01}=+1$.

[27] S. Pokorski, Gauge Field Theories, Cambridge Monographs in Mathematical Physics (Cambridge University Press, Cambridge, England, 2000), Chap. 13. The convention is $\epsilon^{0123}=-1$.

[28] J. Alfaro, A. A. Andrianov, M. Cambiaso, P. Giacconi, and R. Soldati, Bare and induced Lorentz and CPT invariance violations in QED, Int. J. Mod. Phys. A 25, 3271 (2010).

[29] K. Fujikawa, Path-Integral Measure for Gauge-Invariant Fermion Theories, Phys. Rev. Lett. 42, 1195 (1979); Phys. Rev. D 21, 2848 (1980).

[30] J. Alfaro, L. F. Urrutia, and J. Vergara, Extended definition of the regulated jacobian in the path integral calculation of anomalies, Phys. Lett. B 202, 121 (1988). 